\title{
'||||||||||||||||||||||||||||||||||||||||||||||||||||||||||||||||||||.
}

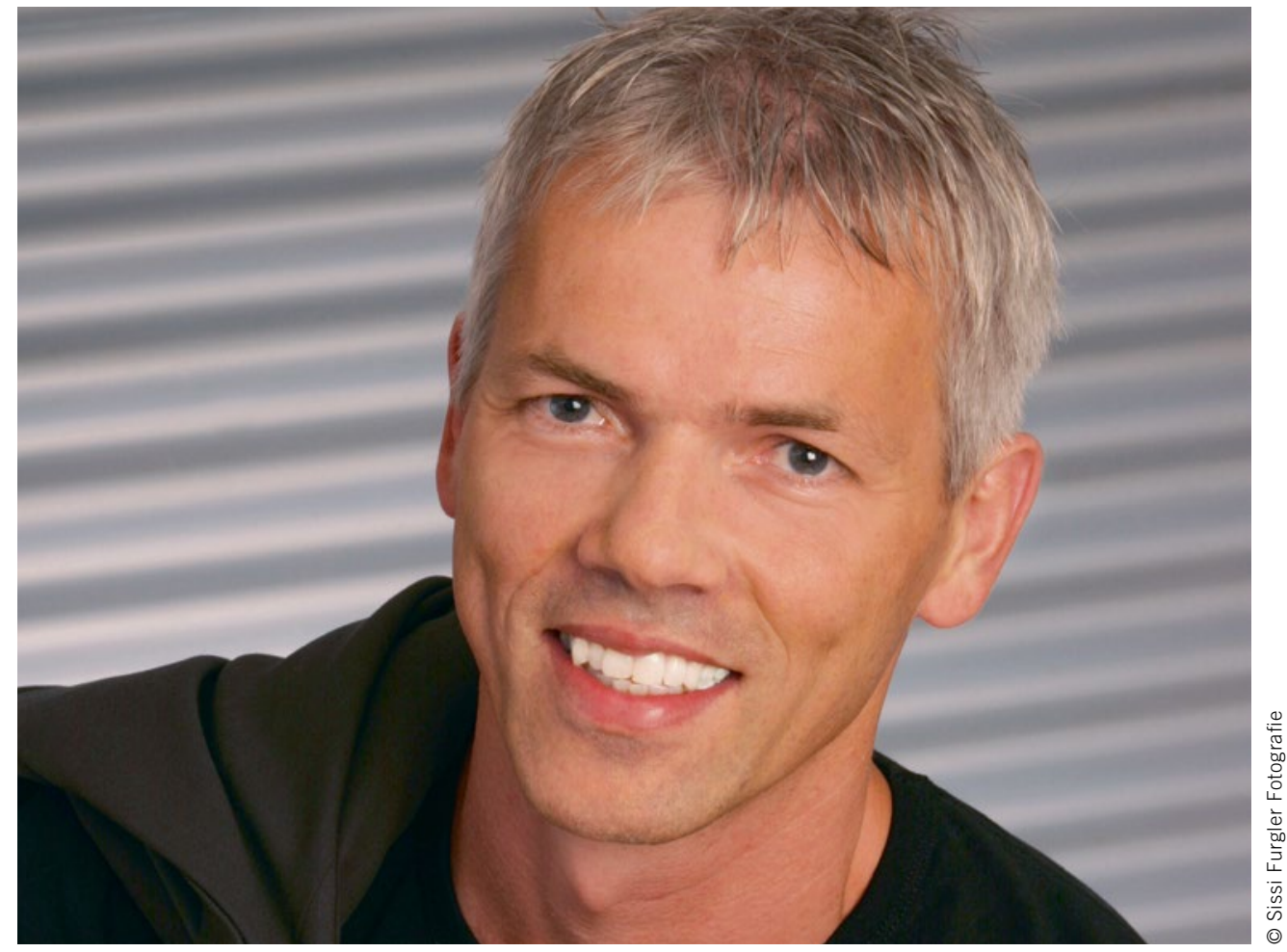

Prof. Dr. Andreas Wimmer Professor at the Institute of Internal Combustion Engines at the Graz University of Technology and CEO of the Large Engines Competence Center (LEC) in Graz (Austria)

\section{Gas Engines - The Outstanding Potentials}

High and medium speed diesels are setting efficiency records and increasing power density when closer parity between the key aspects of large Otto and Diesel engines would assist their interchangeability and the development of dual-fuel engines. The Large Engines Competence Center at the Graz University of Technology is meeting this challenge with research into gas and dual-fuel engine performance involving 22 partners who together represent a spectrum of cutting-edge technologies.

Aside from efficiency and specific output there is a pressing need to address other gas and dual-fuel engine characteristics perceived as deficits vis-à-vis diesels. They include robustness, fuel flexibility, transient response and methane slip.

To increase gas engine robustness an obvious focus is the combustion chamber. The effective life of spark-plugs is the main limiting factor on maintenance intervals and plug life is strongly linked to mean effective pressure and load. At the same time we are looking at the piston, lube oil including oil coking of the ring pack and overall combustion chamber design. The longevity of cylinder pressure sensors is also vital, since they acquire data with huge implications for advanced control.

Transient performance is especially relevant to gas engines as low emissions, fast-reacting back-up for renewable energy sources. As well as enabling adaptation of the Miller cycle, variable valve timing is an effective tool in several areas of control. It is providing valuable variability for dealing with sudden changes in gas quality without sacrificing efficiency, power and safety. This longstanding gas and dual-fuel engine issue is more acute when gases such as hydrogen and bio-gas are fed into the grid or engines are run on special "non-natural" gases such as blast-furnace and flare gas.

Of course, efficiency and power are also a central concern of the project partners and a core research area is combustion processes. Its scope includes high pressure gas injection, where fuel is introduced into the compressed air close to topdead-centre and ignited by a liquid fuel micro-pilot to create diffusion combustion. This is one very effective way to overcome power density and efficiency limitations imposed by combustion knock.

Finally, although gas and dual-fuel engines have intrinsically low $\mathrm{NO}_{\mathrm{x}}, \mathrm{SO}_{\mathrm{x}}$ and $\mathrm{CO}_{2}$ emissions, methane slip is still an issue. $\mathrm{CH}_{4}$ is difficult to eliminate due to its chemical stability, but promising measures are new catalyst coatings and locating the converter ahead of the turbocharger, where temperatures are higher.

Research into these areas of gas and dual engine technology can to create a broad equivalence between diesel and gas engine performance. 